\title{
Biological Synthesis Of Silver Nanoparticles Using A Marine Bacterial Culture Isolated From The West Coast Of India
}

\author{
Roopa Kakarlapudi', Preeti S Mulage', Seema Sharma' and Meenal Kowshik ${ }^{4}$ \\ ${ }^{12.3}$ Birla Institute of Technology \& Science, Pilani-Goa Campus, Zuarinagar, Goa 403726 \\ "Department of Physics, A N College, Patna 800013
}

\begin{abstract}
Nanotechnology is a highly energized discipline of science and technology. One of the major challenges of nanotechnology is the synthesis of nanomaterials with a wide range of chemical compositions and sizes. Moreover, the ability to scale up synthesis strategies for low-cost, large-scale production is an important factor. Thus, there is a need for developing eco-friendly means of nanoparticle synthesis and the use of microorganisms for this purpose is an emerging area. In this paper, a marine culture of Bacillus sphaericus isolated from the west coast of India has been used to synthesize silver nanoparticles. Bacillus sphaericus is grown in Zobell marine broth and cell lysate prepared by suspending the cells in distilled water. When silver ions are added to the cell lysate, they are reduced to silver nanopartides. The time required for silver reduction is drastically reduced if the solution is exposed to light. Optical abscrption, $X$-ray diffraction, TEM, and energy dispersive analysis of $X$-rays showed that the silver particles were in the nanoregime. Reduction of silver appears to be mediated by non-specific cellular constituents. These findings open up several new possibilities of obtaining silver nanoparticles in an economical and environmentally acceptable manner, on alarge scale.
\end{abstract}

Key words:

\section{INTRODUCTION}

Synthesis of nanoparticles has been a challenge for many years because of their novel properties that differ markedly from those seen in the bulk scale. The small size, surface tailorability, improved solubility and multifunctionality of nanoparticles open many new research avenues [1]. The development of reliable experimental protocols for the synthesis of nanoparticles over a range of chemical compositions, sizes and high monodispersity is one of the challenging issues in current nanotechnology [2]. There is a growing need to develop environmentally friendly nanoparticles synthesis processes that do not use toxic chemicals in a synthesis protocol. Many organisms, both uni-cellular and multi-cellular are known to produce inorganic materials either intra- or extra-cellularly [3]. Work in this field is progressing rapidly with many recent findings including extra-cellular synthesis of Magnetite using Fusarium oxysporum and Verticillium [4], silica \& titania particles [5] and Ferroelectric Barium Titanate nano-particles [6] using Fusarium Oxysporum.

Microorganisms which tolerate heavy exposure to metal ions can be used in the synthesis of nanoscale materials. The advantages of the biological method are that it is eco-friendly and also doesn't require extreme temperature and pressure conditions. However, it is a comparatively slow process and a lot of work needs to be done in the direction of improving yields. The strategies to be undertaken in order to overcome this problem would be screening of various environments to find microorganisms with greater efficiency of metal conversion, identifying the protein involved in metal transformation, and studying the logistics of invitro metal transformation etc.

The exact reaction mechanism leading to the formation of metal nanoparticles by microorganisms is not yet fully understood. The formation of metal nanoparticles can be either intra-cellular or extra-cellular. In the intracellular formation, it is speculated that the metal ions are trapped on the surface of the cells, possibly via electrostatic interaction between the metal ions and the negatively charged carboxylate groups in proteins present in the cell walls. Thereafter, the metal ions are reduced by enzymes present in the cell wall leading to the formation of metal nuclei which subsequently grow by further reduction of metal ions and accumulation on these nuclei. It is possible that some metal ions diffuse through the cell wall and are reduced by enzymes present on the cytoplasmic membrane and within the cytoplasm. From the recovery point of view, it would be difficult to harvest the metal nanoparticles formed within the biomass. It would be far more practical if the metal ions exposed to the bacteria could be reduced outside the biomass, leading to the formations of metal.nanoparticles in the solution $[3,7]$. During extracellular synthesis of nanoparticles it is reported that the fungus when exposed to metal ions releases enzymes that reduce the metal ions to yield highly stable nanoparticles in the solution. Also there are proteins secreted by the micro-organism in the solution, which act as capping agents and bind around the metal particles thus preventing agglomeration [7]. However from literature, it is seen that monodispersity is more for nanoparticles which have been synthesized intracellularly 
as compared to extracellularly synthesized nanoparticles $[8,9,10]$.

Microorganisms develop numerous special adaptations to survive in toxiclextreme habitats which include new mechanisms of regulating intracellular environment and metabolism. In the case of bacteria most metal ions are toxic and therefore, reduction of the ions or formation of water insoluble complexes is a defense mechanism developed by them to overcome such toxicity. Micro-organisms exposed to harsh conditions of highly concentrated industrial effluents including heavy metals, radio nucleotides and organic xenobiotics have an increased resistivity to the effect of these toxic pollutants. Metal pollution is common in waters impacted by human activities. Thus, novel bacterial strains can be isolated from such ecological niches containing heavy metal compounds.

During our studies on screening marine environments in order to search for microorganisms with higher efficiency of metal conversion we have been able to isolate a marine culture of Bacillus sphaericus from the west coast of India which is able to synthesize silver nanoparticles extracellularly. This is the first report of silver nanoparticle synthesis using a marine microorganism.

\section{EXPERIMENTAL PROCEDURES}

\section{A. Isolation of Metal resistant Microorganisms}

Sea water samples were collected from various sources along the coast of Goa. Dilutions were prepared and plated on Zobell marine agar plates. The plates were incubated at room temperature for 24 to 48 hours. Distinct looking colonies were picked up and checked for their silver resistance. This was done by spot inoculating the cultures on increasing concentrations of silver as silver nitrate. The various silver concentrations used were 25ppm, 50ppm, 75ppm, 100ppm, 125ppm, 150ppm, $175 \mathrm{ppm}, 200 \mathrm{ppm}, 300 \mathrm{ppm}$ and $400 \mathrm{ppm}$. In all the experiments the silver salt used was silver nitrate. Cultures that could tolerate up to $400 \mathrm{ppm}$ silver were selected forfurther studies.

\section{B. Screening for Synthesis of Ag Nanoparticles}

The selected silver tolerant cultures were inoculated in $100 \mathrm{ml}$ of Zobell marine medium and incubated at room temperature $(110 \mathrm{rpm})$ until the absorbance of the cultures was between $0.8-0.9$. Silver at a concentration of 400 ppm was added and the cultures were incubated further. Cell free supernatant was obtained by centrifuging (Eppendorf 5810R) the cultures at $8000 \mathrm{rpm}$ for 10 minutes at $150 \mathrm{C}$. The absorbance scan of the supernatants was recorded from $200-800 \mathrm{~nm}$ to look for the absorbance peak characteristic of nanosilver. The culture exhibiting the best peak was selected for further studies.

\section{Optimization of Method of Synthesis}

For optimization of phase of silver addition for maximum production the experimentwas run in three sets. In the first set silver was added to the cells after they had reached the stationary phase of growth. In the second set, the cells were grown till stationary phase, recovered by centrifugation, suspended in distilled water and $400 \mathrm{ppm}$ silver added to this suspension. For the third set, the stationary phase cells were suspended in water for 24 hours, subsequently the cells were removed by centrifugation at $8000 \mathrm{rpm}$ for 10 minutes and silver was added to the cell free suspension. Formation of silver nanoparticles was monitored by following the absorbance at $430 \mathrm{~nm}$.

\section{Characterization of Silver Nano-particles}

The optical absorbance measurements of the silver nanoparticles suspended in distilled water were taken on a UV visible spectrophotometer (Shimadzu UV 2450) in the range of 200 to $800 \mathrm{~nm}$. XRD measurements of the silver nanoparticles was taken on a Rigaku Miniflex operated ata voltage of $40 \mathrm{kV}$ and a current of $30 \mathrm{~mA}$ using a Cu-Ka radiation of wavelength $1.5408 \mathrm{~A}$. Transmission electron microscopy (TEM) was performed using a Jeol JEM-1011 electron microscope working at $80 \mathrm{KeV}$ acceleration. $5 \mu \mathrm{l}$ of silver nanoparticles solution was drop-casted on carbon coated copper grids. Scanning Electron Microscopy and energy dispersive analysis of X-rays (SEM-EDAX) of the silver nanoparticle powder was carried out on a JEOL JSM $6360 \mathrm{LV}$. The accelerating voltage used was $5 \mathrm{kV}$

\section{RESULTS AND DISCUSSION}

Screening of marine cultures for silver resistance and subsequent selection of the cultures based on ability to synthesize silver nanoparticles yielded us a culture of Bacillus sphaericus which was able to synthesize silver nanoparticles. This culture could tolerate upto $400 \mathrm{ppm}$ silver which was added to the medium in the form of silver nitrate. Reduction was obtained in all three sets, viz. when silver nitrate was added to stationary phase cells, to the culture suspended in water and to the cell-free suspension. Because recovery of the silver nanoparticles was easier from the third set it was decided to follow this protocol for further synthesis. Since reduction of silver was being carried out in the absence of culture medium the possibility of non specific reduction by the medium constituents was ruled out. An interesting observation was that the time required for silver reduction could be drastically reduced 


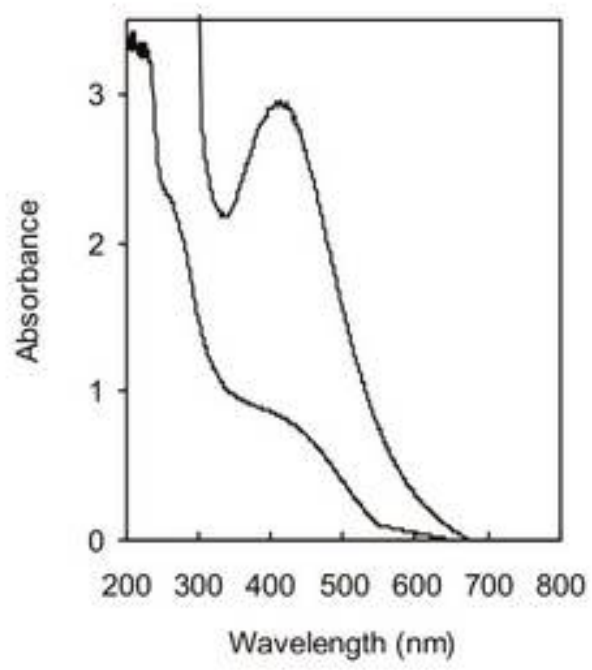

Fig. 1. Absorbance scan of silver nanoparticles formed after 15 minutes of silver salt addition

(a) In presence of light (b) In absence of light

from about 10 hours to 15 minutes by carrying out the synthesis in presence of light and by constant stirring. This overcomes a major drawback of the biological synthesis routes which are known to be slow. The time required for complete reduction of silver ions using bacteria and fungi has been reported to be in the range of 24 to 120 hours [9].

The absorbance scan of the silver nanoparticles exhibited a plasmon peak at $420 \mathrm{~nm}$ which is characteristic of monodispersed $\mathrm{Ag}$ (Fig 1). Plasmon Resonance Nanoparticles are metallic (silver or gold) Nanoparticles which scatter optical light elastically with remarkable efficiency because of a collective resonance of the conduction electrons in the metal (i.e., the surface plasmon resonance). The magnitude, peak wavelength, and spectral bandwidth of the plasmon resonance associated with a nanoparticle are dependent on the particle's size, shape, and material composition, as well as the local environment. From Fig. 1 we can see that the synthesis can be completed within 15 minutes on exposure to light. These particles were found to be stable in solution even on storage upto 1 month with no change in absorbance maxima. In powder form we have stored the silver nanoparticles for over 6 months.

X-ray powder diffractogram of the biosynthesized nanosilver exhibits Bragg reflections due to (111), (200), (220), (311) and (222) corresponding to foc type bulk silver (Fig. 2). The diffraction peaks are broadened around their base indicating that the the silver particles are in nanosizes. The TEM data obtained by placing the suspension of nanosilver on carbon coated copper grids is shown in Figure 3. The silver nanoparticles are in the size range of $10 \mathrm{~nm}$. The particles smaller than $10 \mathrm{~nm}$ were difficult to resolve with the microscope used in these studies. From the SEM images aggregates of about 0.25 $05 \mu \mathrm{m}$ were observed. This probably indicates that the 10 $\mathrm{nm}$ particles are further aggregating to form larger particles. The sample contained about $75 \% \mathrm{Ag}$ as was revealed from the EDAX measurements.

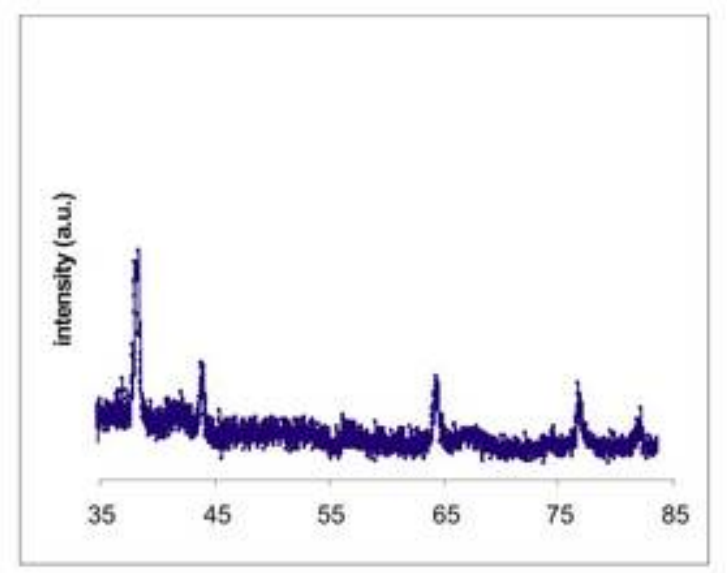

Fig. 2. XRD pattern of Silver nanoparticles.

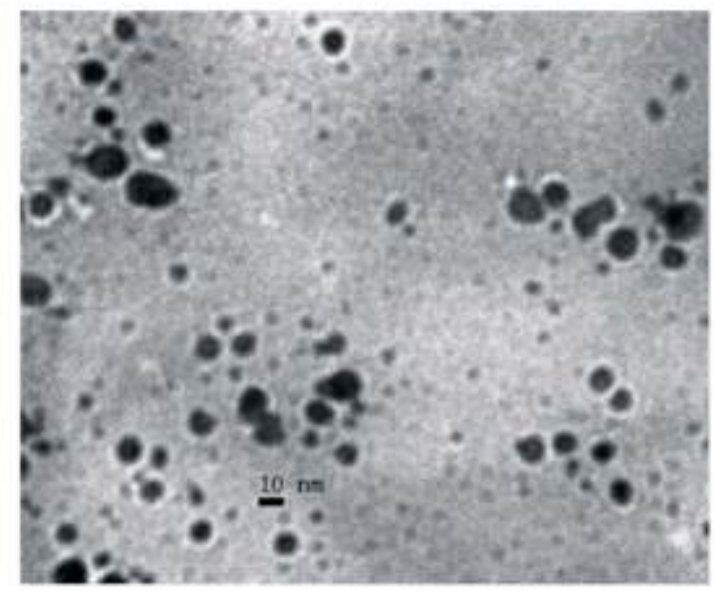

Fig. 3 Transmission Electron Microscopy Micrograph of silver nanoparticles synthesized by Bacillus sphaericus

Although the exact mechanism leading to reduction of silver ions to elemental form is yet to be elucidated, it is possible that certain bio-chemical reducing agents are excreted by the cells on lysis. It is known that Silver cations are highly reactive and tend to bind strongly to electron donor groups containing sulphur, oxygen or nitrogen. Such a binding with bio-molecules like small proteins could restrict the size of the particles [11]. 
In summary, a brief overview of the use of microorganisms in the biosynthesis of $\mathrm{Ag}$ nanoparticles has been described. In the context of the present drive to develop green technologies in materials' synthesis, this method is a viable alternative to the physical and chemical methods currently in vogue. Extra-cellular secretion of enzymes offers the advantage of obtaining large quantities in a relatively pure state, free from other contaminating proteins associated with the organism. The process can be extended to the synthesis of nanoparticles of different chemical compositions, shapes and sizes by suitable identification of the enzymes secreted by the micro-organisms.

\section{ACKNOWLEDGMENT}

We thank Dr Rahul Mohan, NCAOR, Goa for helping us with the SEM-EDAX

Measurements and Dr N Sandhyarani for the TEM measurements

\section{REFERENCES}

[1] S. E. McNeil, "Nanotechnology for the biologist", Journal of Leukocyte Biology, Vol. 78, pp. 585-594, 2005

[2] Mandal D., Bolander M. E., Mukhopadhyay D., Sarkar G. And Mukherjee P. "The use of microorganisms for the formation of metal Nanoparticles and their application", Appl Microbiol Biotechnol, Vol. $69(5), p p .485-92,2006$

[3] A. Ahmad, S. Senapati, R Kumar and M. Sastry, "Extracellular biosynthesis of monodisperse Gold nanoparticles by novel Extremophilic Actinomycete, Thermomonospora sp.", Langmuir, Vol. 19, pp. 8,2003
4] A. Bharde, D. Rautaray, V. Bansal, A. Ahmad, I. Sarkar, S. M. Yusuf, M. Sanyal and M. Sastry, "Extracellular biosynthesis of magnetite using Fungi", Small, Vol. 2, pp. 135-141, 2006

[5] V. Bansal, D. Rautaray, A. Bharde, K. Ahire, A. Sanyal, A. Ahmad and M. Sastry, "Fungus mediated biosynthesis of Silica and Titania particles", J. Mater. Chem., Vol. 15, pp. 2583-2589, 2005

[6] V. Bansal, P. Podar, A. Ahmad and M. Sastry, "Room temperature bio-synthesis of Ferroelectric Barium Titanate nanoparticles", J. Am. Chem Soc, Vol. 128, pp. 11958-11963,2006

[7] Mukherjee P., Senapati S., Mandal D., Ahmad A., Khan M. I., Kumar R., Sastry M., "Extracellular synthesis of gold nanoparticles by the fungus Fusarium oxysporum. ', Chem. Biochem., Vol. 3, pp. $461-463,2002$

[8] M. Kowshik, S. Ashtaputre, S. Kharrazi, W. Vogel, J. Urban, S. K. Kulkarni and K. M. Paknikar, "Extracellular synthesis of Silver nanoparticles by silver tolerant yeast strain MKY3", Nanotechnology, Vol. 14, pp. $95-100,2003$

[9] Kowshik M., Vogel W., Urban J., Kulkarni S. K. and Paknikar K. M., "Microbial synthesis of semiconductor PbS nanocrystallites", Adv. Mater., Vol. 14, pp. 815,2002

[10] Kowshik M., Deshmukh N., Vogel W., Urban J., Kulkarni S. K. And Paknikar K. M., "Microbial synthesis of semiconductor CdS nanoparticles, their charecterisation and their use in the fabrication of an idealdiode", Biotech. Bioeng., Vol. 78, pp. 583, 2002

[11] Trevors JT, 1987, Enz. Microbiol. Tech., 9, 331 